\title{
Uso da adubação verde na recuperação de solos degradados por mineração na floresta amazônica
}

\author{
Regina Márcia Longo ( $\left.{ }^{1 *}\right)$; Admilson Írio Ribeiro (²); Wanderley José de Melo $\left(^{3}\right)$ \\ (') Faculdade de Engenharia Ambiental, PUC-CAMPINAS. E-mail:rmlongo@uol.com.br (*) Autora correspondente. \\ (2) Faculdade de Engenharia Ambiental, UNESP-Sorocaba.E-mail:admilson.ribeiro@uol.com.br \\ (3) Faculdade de Ciências Agronômicas e Veterinárias, UNESP, Via de acesso Prof. Paulo Donato Castellane, Km 5, 14884-900 \\ Jaboticabal (SP). E-mail:wjmelo@fcav.unesp.br
}

Recebido: 10/mar./2010; Aceito: 11/jun./2010

\begin{abstract}
Resumo
O presente trabalho teve como objetivo avaliar: (a) Uso de leguminosas e gramíneas utilizadas na adubação verde; (b) Incorporação de matéria orgânica pela adição de compostos orgânicos e (c) Efeitos da adubação química e calagem na recuperação de áreas degradadas pela mineração de cassiterita na FLONA do Jamari (RO). O experimento foi instalado em dezembro de 1999. O delineamento experimental adotado foi em blocos casualizados com 24 tratamentos no esquema fatorial 6 X 4 , constando de seis tipos de coberturas vegetais (Brachiaria humidicula (Braquiária), Cajanus cajan (Feijão guandu), Mucuma aterrima (Mucuna preta), Cajanus cajan (Feijão guandu) + Brachiaria humidicula (Braquiária); Mucuma aterrima (Mucuna preta)+ Brachiaria humidicula (Braquiária) e solo nu e quatro tipos de adubação na cova (NPK 10-10-10; 50\% rejeito + 50\% composto; 50\% rejeito + 50\% composto + NPK 10-10-10; 50\% rejeito + 50\% composto + NPK 10-10-10 + calcário dolomítico). As amostras de solo foram coletadas superficialmente $(0-20 \mathrm{~cm})$ para fins de análises químicas e físicas, 15 meses após 0 plantio, logo após as chuvas. Os resultados foram submetidos à análise de variância $(p<0,05)$ e, nos casos em que o teste F foi significativo, aplicou-se o teste Tukey para a comparação das médias de seis repetições Apenas nas características químicas foram observadas diferenças significativas entre os tratamentos. As adubações química e orgânica e a calagem contribuíram para essas diferenças, e os tratamentos que receberam calcário proporcionaram como esperado aumento no pH, nos teores de Ca, Mg, na CTC, SB e V\% e diminuição no H+Al quando comparados aos demais. Porém, quando se analisa o teor de matéria orgânica e os parâmetros físicos estudados não houve diferenças entre os tratamentos, mesmo naqueles onde a adubação foi intensiva associada ao plantio de adubos verde e a gramíneas indicando que as melhorias nos atributos físicos do solo em áreas de deposição de rejeito deve ocorrer de forma mais lenta.
\end{abstract}

Palavras-chave: solo degradado, fertilidade, leguminosas.

\section{Use of green fertilizing in the recovery of soils degraded by mining in the amazon forest}

\section{Abstract}

This work had the goal of evaluating (a) The use of legumes and grasses employed in green fertilizing; (b)The incorporation of organic matter through the addition of organic compounds and (c) The effects of chemical fertilizing and lime application in the recovery of areas degraded by tin mining in the Jamari National Forest (RO). The experiment was set up in December, 1999. The experimental design adopted consisted of casualized blocks with 24 treatments according to a $6 \times 4$ factorial scheme, composed by six types of plantcoverage (Brachiaria humidicula, Cajanus cajan, Mucuna aterrima, Cajanus cajan + Brachiaria humidicula, Mucuna aterrima + Brachiaria humidicula, and bare soil), and four types of fertilizer in the pit (NPK 10-10-10; 50\% tailing + 50\% compound; 50\% tailing + 50\% compound + NPK 10-10-10; 50\% tailing + 50\% compound + NPK 10-10-10 + dolomite lime. The soil samples were collected superficially $(0-20 \mathrm{~cm})$ and used for chemical and physical analyses in 144 installments, 15 months after planting, right after the rains fall periods. The results were submitted to analysis of variance $(p<0,05)$ when test $F$ proved significant, the Tukey test was applied for comparison of the averages of six repetitions. The chemical characteristics were the only ones to present significant differences between treatments. The chemical and organic fertilizing, as well as the lime application, contributed for these differences, and the treatments that received lime presented a series of improvements in soil, such as an increase in $\mathrm{pH}$, in the levels of $\mathrm{Ca}$, $\mathrm{Mg}$, in $\mathrm{CTC}, \mathrm{SB}$ and $\mathrm{V} \%$ and a decrease in $\mathrm{H}+\mathrm{Al}$ when compared to the others. However, when the level of organic matter was analyzed, the physical parameters and the biological aspects studied did not present differences between treatments, even in those where fertilizing was intensively associated to the planting of green manure and the introduction of forest species, indicating that the improvements in the physical attributes of the soil in tailing areas must happen at a slower pace.

Key words: degraded soil, fertility, legumes. 


\section{INTRODUÇÃO}

Um aspecto fundamental na recuperação de áreas degradadas pela mineração é o conhecimento do solo onde essa recuperação será realizada. Os procedimentos específicos na recuperação dessas áreas dependem essencialmente das propriedades físicas, químicas e mineralógicas do solo que deverá ter condiçôes para o adequado desenvolvimento das plantas (Fontes, 1991). Desta forma, para que os projetos de recuperação de áreas degradadas obtenham sucesso é necessário caracterizar o solo em que se vai implantar a revegetação para definição das açốes iniciais ou dos tratamentos que seráo aplicados.

Em muitas áreas degradadas o que se nota não é mais solo, mas uma matriz de material mineral, de pobre estrutura física, pouca ou nenhuma matéria orgânica e quantidade muito pequena de nutrientes para as plantas. Se uma cobertura vegetal puder ser estabelecida, iniciar-se-á o processo de formação do solo por meio das alteraçóes químicas, físicas e biológicas do próprio solo, promovidas pela atividade rizosférica das raízes. A falta de matéria orgânica constitui um dos principais problemas da recuperaçáo dessas áreas degradadas. O armazenamento e a reutilização da camada fértil do solo produzem excelentes resultados, porém essa é uma técnica dispendiosa e difícil, em virtude da pouca profundidade dessa camada nos solos tropicais ou da acentuada declividade do solo em muitas minas. O plantio de leguminosas arbóreas de rápido crescimento, capazes de formar em curto prazo a cobertura vegetal, tem sido uma prática viável. A maioria dessas espécies, abundantes e diversificadas no Brasil, produz grandes quantidades de biomassa e seu aporte de matéria orgânica folhosa contribui significativamente ao incremento de carbono e nutrientes ao solo (GRIFFITH et al., 1996).

Outro aspecto de grande importância a ser considerado na recuperação de áreas degradadas pela mineração relaciona-se às características físicas dos solos. De acordo com Silva e Ribeiro (1992) mesmo com o uso de fertilizantes, variedades adaptadas e controle de pragas e doenças, boas produtividades não serão atingidas se houver degradação das características físicas do solo.

Segundo Griffith (1980), a única maneira de se mitigar os impactos no solo, causados pela mineração, é por meio do restabelecimento de uma cobertura vegetal perene sobre o local minerado em curto tempo, posto que o processo de sucessão é lento enquanto a erosão é imediata e acelerada, perpetuando o processo de degradaçáo.

Pelas alteraçôes impostas às características físicas, químicas e biológicas do solo, o processo de revegetação é dificultado, sobretudo, em decorrência de um ou mais dos problemas tais como: níveis tóxicos de metais; altas concentraçôes de sais solúveis; valores extremos de $\mathrm{pH}$ (altos ou baixos demais, dependendo do tipo de rejeito liberado); deficiências nutricionais; baixa taxa de infiltração de água; alta temperatura na superfície, baixa capacidade de troca de cátions dos solos; atividade biológica restrita; e baixa retenção de água (Williams et al., 1990; Silva, 1993). Esses fatores contribuem para a degradação do solo, que segundo Oliveira (1995), ocasiona várias consequências como a compactação do solo, crosta, baixa capacidade de infiltração de águas, problemas relacionados à aeração, erosão acelerada, perda de fertilidade natural do solo e alteraçóes nas populaçóes microbiológicas do solo.

$\mathrm{O}$ presente trabalho teve como objetivo avaliar o uso de leguminosas e gramíneas de rápido crescimento; a incorporação de matéria orgânica pela adição de compostos orgânicos e os efeitos da adubação química e calagem na recuperação de áreas degradadas pela mineração de cassiterita na FLONA do Jamari/RO, avaliando aspectos químicos e físicos do solo em área de rejeito arenoso.

\section{MATERIAL E MÉTODOS}

A Floresta Nacional do Jamari, administrada pelo Instituto Brasileiro do Meio Ambiente e Recursos Minerais Renováveis - IBAMA, está situada a $90 \mathrm{~km}$ da cidade de Porto Velho (RO), pela Br-364, rumo a Cuiabá (MT) (Figura 1). Essa área compóe-se de aproximadamente 225.000 ha, das quais $90 \%$ estão cobertas por Floresta Tropical Aberta, onde são encontradas espécies de alto valor comercial para exploraçáo de madeira, sendo também rica em minérios (RADAMBRAsil, 1978).

Os solos são predominantemente da classe Latossolo Vermelho-Amarelo álico textura argilosa e Latossolo Amarelo álico textura argilosa (EMBrapa, 1999). O clima da região é quente e úmido, com temperaturas médias de $24^{\circ} \mathrm{C}$, e precipitação pluvial anual de $2550 \mathrm{~mm}$, tendo seus máximos de dezembro a março. A umidade relativa fica em torno de $80 \%$ a $85 \%$, havendo uma estação seca bem definida, com seu período mais crítico de julho a agosto.

Desde o início da década de 70, a mineraçáo Jacundá vinha explorando cassiterita na FLONA do Jamari em Rondônia. Esta atividade criou áreas degradadas, cuja recuperaçáo vem sendo realizada.

O experimento foi instalado entre dezembro de 1999 e janeiro de 2000, no local denominado de Serra da Onça (SO), coordenadas UTM, zona 20, 496.721E e 8.987.202N, em uma área de deposição de rejeito arenoso com aproximadamente 20 metros de profundidade. As características físicas e químicas do rejeito são: $\mathrm{pH}=$ $4,0\left(\mathrm{CaCl}_{2}\right)$, matéria orgânica $=7,0 \mathrm{~g} \mathrm{dm}^{-3}$, fósforo $=2,0$ $\mathrm{g} \mathrm{dm}^{-3}$, potássio $=0,3 \mathrm{mmolc} \mathrm{dm}^{-3}$, cálcio $=1,0 \mathrm{mmolc}$ $\mathrm{dm}^{-3}$, magnésio $=1,0$ mmolc dm ${ }^{-3}, \mathrm{H}+\mathrm{Al}=15,0$ mmolc $\mathrm{dm}^{-3}$, soma de base $=2,6 \mathrm{mmolc} \mathrm{dm}^{-3}$, capacidade de troca catiônica $=18 \mathrm{mmolc} \mathrm{dm}^{-3}, \mathrm{~V} \%=18,0 \mathrm{mmolc} \mathrm{dm}^{-3}$, areia $=732,0 \mathrm{~g} \mathrm{~kg}^{-1}$, silte $=140,0 \mathrm{~g} \mathrm{~kg}^{-1}$ e areia $=124,0 \mathrm{~g} \mathrm{~kg}^{-1}$.

$\mathrm{O}$ delineamento experimental adotado foi em blocos casualizados com 24 tratamentos no esquema fatorial 
$6 \mathrm{X} 4$, constando de seis tipos de coberturas vegetais (Brachiaria humidicula (Braquiária), Cajanus cajan ( Feijão guandu), Mucuma aterrima (Mucuna preta), Cajanus cajan ( Feijão guandu) + Brachiaria humidicula (Braquiária); Mucuma aterrima (Mucuna preta)+ Brachiaria humidicula (Braquiária) e solo nu) e quatro tipos de adubação química e orgânica na cova: A1) rejeito + NPK 10-10-10; A2) 50\% rejeito + 50\% composto orgânico (serragem + esterco bovino, na proporção de 3:1); A3) $50 \%$ rejeito + 50\% composto + NPK 10-1010; A4) $50 \%$ rejeito + 50\% composto + NPK 10-10-10 + calcário dolomítico, com 6 repetiçôes, totalizando 144 parcelas correspondendo a uma área experimental de 1,5 ha. $\mathrm{O}$ tratamento com calcário recebeu $2 \mathrm{t} \mathrm{ha}^{-1}$ desse produto em área total, o que corresponde a uma dose de $20 \mathrm{~kg}$ de calcário por parcela, de acordo com o tratamento estabelecido. A mucuna preta e o feijão guandu foram semeados em linha, com espaçamento de $0,50 \mathrm{~m}$ entre plantas e densidade de semeadura de 60 e $80 \mathrm{~kg} \mathrm{ha}^{-1}$ de sementes respectivamente. A braquiária foi semeada a lanço, adubada da mesma forma e com densidade de plantio de $60 \mathrm{~kg} \mathrm{ha}^{-1}$ de sementes.

As amostras de solos foram coletadas para fins de análises químicas e físicas 15 meses após a semeadura $(15 / 12 / 2000)$ na profundidade de $0-0,20 \mathrm{~m}$. A análise granulométrica foi realizada pelo método da pipeta, descrita em Kiehl (1979) em amostras de TFSA. Para a densidade do solo a amostra foi obtida com o uso do anel volumétrico (50 $\mathrm{m}^{3}$ de volume) e com sua estrutura natural deformada, transferiu-se para um recipiente, no próprio campo, o conteúdo do anel. No laboratório, a amostra foi pesada e seca em estufa a $105-110{ }^{\circ} \mathrm{C}$ por 24 horas e em seguida pesada novamente, obtendo-se assim a densidade do solo das amostras (KieHL, 1979). A densidade de partículas foi determinada pelo método do Balão volumétrico (KIEHL, 1979). Utilizando-se os dados obtidos com a densidade do solo e de partícula, determinouse a porosidade total das amostras.

Para as análises de fertilidade foram retiradas 10 amostras simples por parcela, totalizando 144 amostras compostas, sendo retiradas na profundidade de $0-0,20 \mathrm{~m}$ em seguida foram secas e peneiradas obtendo-se amostras de terra fina seca ao ar (TFSA).

Nessas amostras determinaram-se: os teores de argila, silte e areia total, o $\mathrm{pH}\left(\mathrm{CaCl}_{2}\right)$; o teor de matéria orgânica por oxidaçáo pelo $\mathrm{H}_{2} \mathrm{SO}_{4}$ e por quantificaçáo colorimétrica; o fósforo disponível (P) e os teores de potássio (K), o cálcio $(\mathrm{Ca})$ e o magnésio $(\mathrm{Mg})$, extraídos pela resina de troca iônica, sendo o P quantificado por colorimetria e o $\mathrm{K}, \mathrm{Ca}$ e $\mathrm{Mg}$ por espectrofotometria de absorção atômica; o teor de alumínio (Al) extraído pelo $\mathrm{KCl} 1 \mathrm{~N}$ e determinado por titulação ácido-base; os valores de $\mathrm{T}$ = capacidade de troca de cátions e $\mathrm{V}$ = saturação por bases, foram obtidos por cálculo. Uma descrição, mais detalhada dos métodos analíticos empregados pode ser vista em RAIJ et al. (1987).
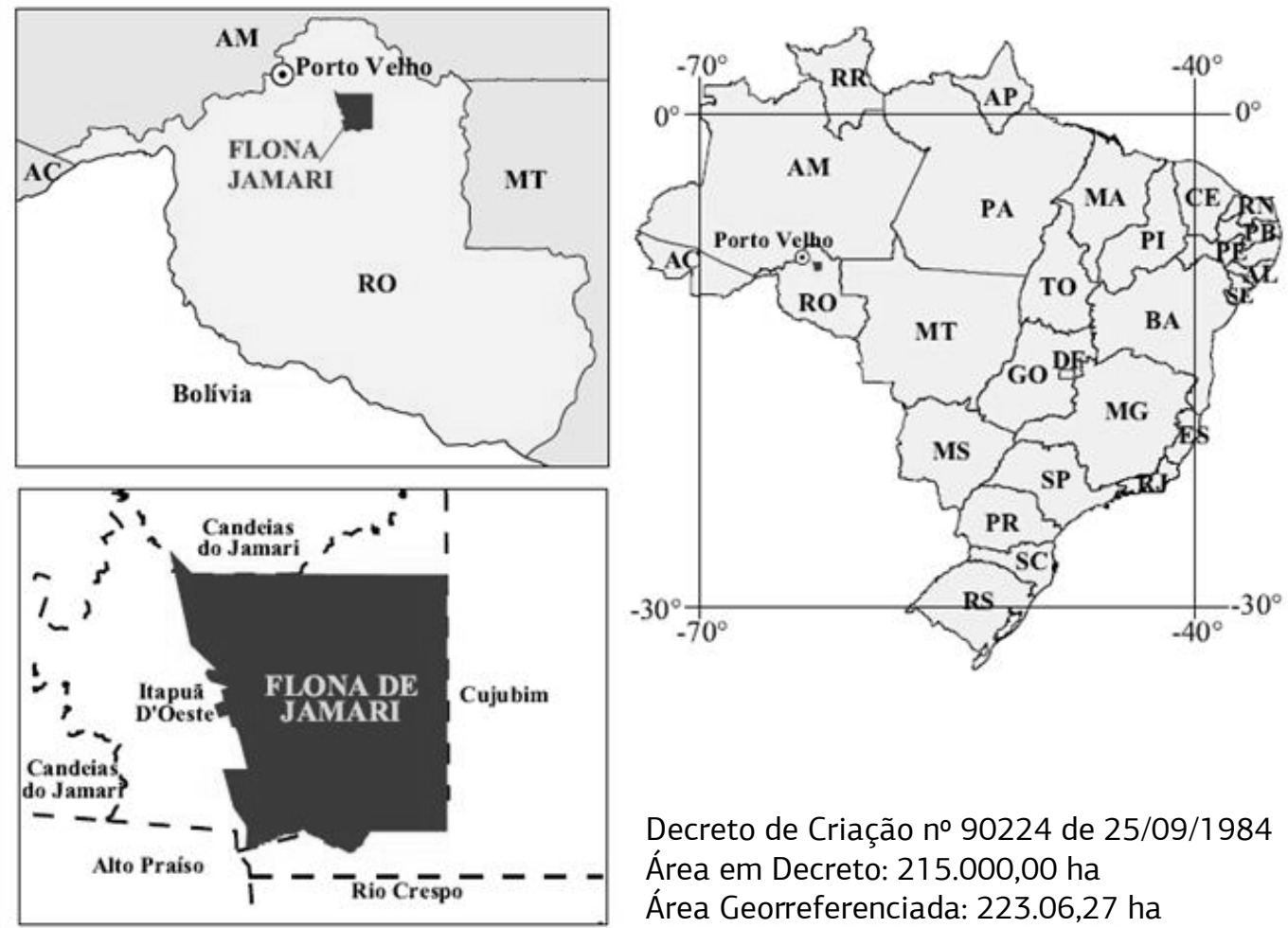

Decreto de Criação no 90224 de 25/09/1984

Área em Decreto: 215.000,00 ha

Área Georreferenciada: 223.06,27 ha

Figura 1. Localização da FLONA do Jamari (RO). (Fonte: IBAMA) 
A análise da cobertura verde, formada pelas leguminosas (feijão guandu e mucuna preta), gramíneas (braquiaria) e pelo surgimento espontâneo de espécies que atuam na colonização inicial do terreno, foi feita adotando-se o sistema de avaliação com notas (0-10) a cada uma das parcelas plantadas, considerando-se a porcentagem de área coberta. As notas finais foram obtidas pela média das notas parciais ministradas por 6 pessoas diferentes. Optou-se por esta avaliação, pois ela não provocou a retirada dos indivíduos avaliados que, permanecendo no terreno, puderam brotar novamente no ano seguinte, promovendo maior acúmulo de matéria orgânica e a ciclagem de nutrientes, que foi o objetivo principal deste trabalho.

Os resultados obtidos foram submetidos à análise de variância $(p<0,05)$ e, nos casos em que o teste $F$ foi significativo, aplicou-se o teste Tukey para a comparação das médias de seis repetiçôes (BanZatto e Kronka, 1992).

\section{RESULTADOS E DISCUSSÃO}

Os valores de $\mathrm{pH}$, matéria orgânica, fósforo, potássio, cálcio, magnésio, $\mathrm{H}+\mathrm{Al}$, capacidade de troca catiônica, Soma de Bases e porcentagem de saturação por bases (Tabelas 1 a 3).

Em relação ao $\mathrm{pH}$ observa-se pela tabela 2 que houve diferença significativa entre os tratamentos e, como era de se esperar, o tratamento que recebeu aplicação de calcário os valores de $\mathrm{pH}$ foram superiores aos demais em todas as coberturas estudadas. No tratamento que recebeu calcário os maiores valores de $\mathrm{pH}$ foram anotados para o guandu, mucuna + braquiaria e solo nu.

Longo et al. (2005), em estudos realizados nos solos dessas áreas observaram que o processo de extração de cassiterita promoveu alteraçôes significativas, sendo os atributos como o $\mathrm{pH}$, teores de matéria orgânica, $\mathrm{P}$, $\mathrm{K}, \mathrm{V} \%, \mathrm{CTC}, \mathrm{H}+\mathrm{Al}$ os mais alterados pelo processo de supressão da vegetação original e extração do minério.

Tabela 1. Valores de $\mathrm{pH}$ em rejeito arenoso com diferentes coberturas vegetal e tipos de adubação

\begin{tabular}{|c|c|c|c|c|c|c|}
\hline \multirow{2}{*}{ Adubação } & \multicolumn{6}{|c|}{ Cobertura vegetal } \\
\hline & $\mathbf{S}$ & G & M & B & $G+B$ & $M+B$ \\
\hline A1 & 4,88Ba & 4,97Ba & 4,90 Aa & $5,00 \mathrm{Aa}$ & $5,18 \mathrm{Ba}$ & $5,08 \mathrm{Ba}$ \\
\hline A3 & 5,25Ba & $5,05 \mathrm{Ba}$ & $5,13 \mathrm{Aa}$ & 4,93 Aa & 4,90 Ba & $5,03 \mathrm{Ba}$ \\
\hline A4 & $6,35 \mathrm{Aa}$ & 6,38Aa & $5,35 \mathrm{Ab}$ & $5,15 \mathrm{Ab}$ & $5,83 \mathrm{Ab}$ & 6,35 Aa \\
\hline
\end{tabular}

$\mathrm{S}=$ solo nu; $\mathrm{G}=$ feijăo guandu; $\mathrm{M}=$ mucuna; $\mathrm{B}=$ braquiária; $\mathrm{G}+\mathrm{B}=$ guandu + braquiária; $\mathrm{M}+\mathrm{B}=$ mucuna + braquiária. $\mathrm{A} 1=$ adubaçăo química $(\mathrm{NPK}) ; \mathrm{A} 2$ = adubação orgânica (composto orgânico); A3 = adubação orgânica (composto orgânico) + NPK; A4 = adubação orgânica (composto orgânico) + NPK + calagem.

Para cada cobertura, letras minúsculas iguais na linha indicam que as médias não diferiram entre si, pelo teste de Tukey $(\mathrm{p}>0,05)(\mathrm{n}=6)$.

Para cada adubaçáo, letras maiúsculas iguais na coluna indicam que as médias não diferiram entre si, pelo teste de Tukey $(\mathrm{p}>0,05)(\mathrm{n}=6)$

Tabela 2. Teores de Ca, Mg e P e Matéria orgânica em rejeito arenoso com diferentes coberturas vegetal e tipos de adubação

\begin{tabular}{|c|c|c|c|c|c|c|}
\hline \multirow{2}{*}{ Adubação } & \multicolumn{6}{|c|}{$\mathrm{Ca}\left(\mathrm{mmol}_{\mathrm{c}} \mathrm{dm}^{-3}\right)$} \\
\hline & $S$ & G & M & B & $G+B$ & $M+B$ \\
\hline A1 & 2,33Ba & 3,17 Ba & 2,67 Aa & $2,67 \mathrm{Aa}$ & 3,33 Ba & $2,83 \mathrm{Ba}$ \\
\hline A2 & 2,67 Ba & 3,67 Ba & $2,50 \mathrm{Aa}$ & 3,33 Aa & 2,67 Ba & $2,33 \mathrm{Ba}$ \\
\hline A3 & 3,33 Ba & $3,17 \mathrm{Ba}$ & $2,83 \mathrm{Aa}$ & 4,83 Aa & $2,67 \mathrm{Ba}$ & 2,67 Ba \\
\hline \multirow[t]{2}{*}{ A4 } & $9,33 \mathrm{Aa}$ & $8,83 \mathrm{Aa}$ & $5,00 \mathrm{Ab}$ & $4,33 \mathrm{Ab}$ & $8,50 \mathrm{Ab}$ & $8,67 \mathrm{Aa}$ \\
\hline & \multicolumn{6}{|c|}{$\mathrm{Mg}\left(\mathrm{mmol}_{\mathrm{c}} \mathrm{dm}^{-3}\right)$} \\
\hline A1 & $1,00 \mathrm{Ba}$ & $1,83 \mathrm{Ba}$ & $1,17 \mathrm{Aa}$ & 1,17 Ba & $1,50 \mathrm{Ba}$ & $1,17 \mathrm{Ba}$ \\
\hline A2 & $1,00 \mathrm{Ba}$ & $1,83 \mathrm{Ba}$ & $1,17 \mathrm{Aa}$ & $1,67 \mathrm{ABa}$ & $1,17 \mathrm{Ba}$ & $1,17 \mathrm{Ba}$ \\
\hline A3 & $1,50 \mathrm{Ba}$ & 1,33 Ba & $1,50 \mathrm{Aa}$ & $1,83 \mathrm{Aa}$ & $1,00 \mathrm{Ba}$ & $1,50 \mathrm{Ba}$ \\
\hline \multirow[t]{2}{*}{ A4 } & $6,17 \mathrm{Aa}$ & $7,83 \mathrm{Ab}$ & $3,00 \mathrm{Ac}$ & $4,33 \mathrm{Abc}$ & $6,67 \mathrm{Ab}$ & 7,33 Ab \\
\hline & \multicolumn{6}{|c|}{$P\left(\mathrm{mmol}_{\mathrm{c}} \mathrm{dm}^{-3}\right)$} \\
\hline A1 & 2,83Aa & 3,33 Aa & $3,67 \mathrm{Aa}$ & 3,67 Aa & $3,17 \mathrm{Aa}$ & 3,67 Aa \\
\hline A2 & $2,83 \mathrm{Aa}$ & $3,00 \mathrm{Aa}$ & $3,00 \mathrm{Aa}$ & 2,67 Aa & $3,67 \mathrm{Aa}$ & 2,67 Aa \\
\hline A3 & $3,50 \mathrm{Aa}$ & $2,00 \mathrm{Bb}$ & $2,83 \mathrm{Aa}$ & 2,83 Aa & $3,50 \mathrm{Aa}$ & 2,33 Aa \\
\hline \multirow[t]{2}{*}{ A4 } & 1,67 Ba & $2,50 \mathrm{Aa}$ & $2,33 \mathrm{Ba}$ & $2,00 \mathrm{Bb}$ & $2,17 \mathrm{Ba}$ & $2,50 \mathrm{Ba}$ \\
\hline & \multicolumn{6}{|c|}{ Mo $\left(\mathrm{mg} \mathrm{dm} \mathrm{dm}^{-3}\right)$} \\
\hline A1 & 7,4 Aa & $8,5 \mathrm{Aa}$ & 7,5 Aa & $10,2 \mathrm{Aa}$ & 9,5 Aa & $8,5 \mathrm{Aa}$ \\
\hline A2 & 6,8 $\mathrm{Aa}$ & $9,2 \mathrm{Aa}$ & $8,5 \mathrm{Aa}$ & 9,4 Aa & 9,4 Aa & $8,5 \mathrm{Aa}$ \\
\hline A3 & 7,2 Aa & $8,3 \mathrm{Aa}$ & 8,9 Aa & 8,7 Aa & 8,7 Aa & 7,9 Аa \\
\hline A4 & $8,2 \mathrm{Aa}$ & $9,1 \mathrm{Aa}$ & $8,5 \mathrm{Aa}$ & $8,2 \mathrm{Aa}$ & $8,2 \mathrm{Aa}$ & 8,5 Aa \\
\hline
\end{tabular}

$\mathrm{S}=$ solo nu; $\mathrm{G}$ = feijăo guandu; $\mathrm{M}=$ mucuna; $\mathrm{B}=$ braquiaria; $\mathrm{G}+\mathrm{B}=$ guandu + braquiária; $\mathrm{M}+\mathrm{B}=$ mucuna + braquiária. $\mathrm{A} 1=$ adubaçăo química $(\mathrm{NPK}) ; \mathrm{A} 2$ = adubaçăo orgânica (composto orgânico); A3 = adubação orgânica (composto orgânico) + NPK; A4 = adubação orgânica (composto orgânico) + NPK + calagem.

Para cada cobertura, letras minúsculas iguais na linha indicam que as médias năo diferiram entre si, pelo teste de Tukey $(\mathrm{p}>0,05)(\mathrm{n}=6)$.

Para cada adubação, letras maiúsculas iguais na coluna indicam que as médias não diferiram entre si, pelo teste de Tukey $(\mathrm{p}>0,05)(\mathrm{n}=6)$. 
Em relação à matéria orgânica (Tabela 2), nos tratamentos não houve diferenças significativas. Mesmo onde houve a semeadura de adubos verdes náo ocorreram incrementos nos teores de matéria orgânica, demonstrando que os 15 meses decorridos não foram suficientes para promover mudanças no horizonte superficial estudado, sendo necessário um tempo maior para a alteração nos teores. A matéria orgânica é de fundamental importância para o processo de recuperação, visto que esta promove melhorias em atributos físicos, químicos e biológicos do solo, dando sustentabilidade ao sistema a ser recuperado. RAIJ e ZuLLo (1987) destacaram que os teores de matéria orgânica do solo decorrem do equilíbrio de ganhos e perdas; em geral, valores mais altos desse atributo são observados em solos florestais; já em solos construídos, é comum seu baixo teor, o que confirma os resultados avaliados nas áreas estudadas no presente trabalho. Costa e Zocche (2009) encontraram baixos teores de matéria orgânica e fósforo em áreas de mineração, indicando restriçóes ao desenvolvimento de plantas. Resultados semelhantes foram obtidos por SiLva et al (2004).

O cálcio, magnésio e fósforo propiciaram diferenças significativas entre os tratamentos, como pode se observar pela tabela 3. Para o cálcio e o magnésio os maiores valores foram anotados no tratamento que recebeu calcário, exceto o tratamento $\mathrm{A} 3$ na cobertura braquiária; as melhores coberturas foram mucuna, guandu+braquiaria e mucuna+braquiaria para ambos os parâmetros. Para o fósforo, os maiores valores foram verificados na adubação NPK (sem adição de composto e/ou calcário). O P foi mais baixo para todas as coberturas, com exceção para cobertura G (feijão guandu). SANChes (2002) observou que após a adubação fosfatada, o cultivo e a incorporação de mucuna preta e bandarra, houve aumento nos teores de matéria orgânica, nitrogênio total e fósforo em substratos degradados pela mineração de cassiterita.

Ao se comparar esses dados, com os do trabalho de Cochurame e Sanches (1982) que considera P o elemento mais limitante ao crescimento das plantas, com deficiência em $90 \%$ dos solos da regiáo amazônica, seguido pelo $\mathrm{N}$ e pelo $\mathrm{K}$, observa-se certa correspondência. Entretanto, quando essas limitaçôes de natureza química são superadas pela aplicação de calcário, a produtividade das plantas, quando se trata de culturas, passam ser equivalentes às obtidas em solos quimicamente melhores.

De acordo com Raij et al. (1996) para o cultivo de adubos verdes a recomendação é que se eleve o teor de $\mathrm{Mg}$ a um mínimo de $5 \mathrm{mmol}_{\mathrm{c}} \mathrm{dm}^{-3}$, valor que foi atingido em quase todos os tratamentos que receberam calcário, valores observados na tabela 3 .

A capacidade de troca de cátions $(\mathrm{T})$, soma de bases (SB) e V\%, os valores tiveram diferenças significativas entre si (Tabela 3). As maiores tendências e valores ocorreram no tratamento que recebeu adubação química, orgânica e calagem e as melhores coberturas foram solo nu, braquiária, mucuna+braquiária e guandu+braquiária para todos os tratamentos. $\mathrm{O} \mathrm{H}+\mathrm{Al}$ revelou as maiores tendências e valores nos tratamentos onde não foi realizada a calagem, como pode se observar na tabela 4 .

Tabela 3. Teores de T, SB, $\mathrm{H}+\mathrm{Al}\left(\mathrm{mmol}_{\mathrm{c}} \mathrm{dm}^{-3}\right)$ e V\% orgânica em rejeito arenoso com diferentes coberturas vegetal e tipos de adubação

\begin{tabular}{|c|c|c|c|c|c|c|}
\hline \multirow{2}{*}{ Adubação } & \multicolumn{6}{|c|}{$T\left(\mathrm{mmol}_{\mathrm{c}} \mathrm{dm}^{-3}\right)$} \\
\hline & s & G & M & B & $G+B$ & $M+B$ \\
\hline A1 & $14,58 \mathrm{Ba}$ & $16,12 \mathrm{Ba}$ & $14,78 \mathrm{Aa}$ & $14,70 \mathrm{Aa}$ & $16,17 \mathrm{Ba}$ & $14,68 \mathrm{Ba}$ \\
\hline A2 & $15,13 \mathrm{Ba}$ & $16,42 \mathrm{Ba}$ & $15,27 \mathrm{Aa}$ & $16,30 \mathrm{Aa}$ & $15,57 \mathrm{Ba}$ & $15,17 \mathrm{Ba}$ \\
\hline A3 & $15,52 \mathrm{ABa}$ & $15,22 \mathrm{Ba}$ & $15,77 \mathrm{Aa}$ & $18,63 \mathrm{Aa}$ & $15,07 \mathrm{Ba}$ & $15,53 \mathrm{Ba}$ \\
\hline \multirow[t]{2}{*}{ A4 } & $14,92 \mathrm{Aa}$ & $24,72 \mathrm{Aa}$ & $18,02 \mathrm{Ab}$ & 19,83 Aab & $24,15 \mathrm{Aa}$ & $24,17 \mathrm{Aa}$ \\
\hline & \multicolumn{6}{|c|}{$\mathrm{SB}\left(\mathrm{mmol}_{\mathrm{c}} \mathrm{dm}^{-3}\right)$} \\
\hline A1 & $3,92 \mathrm{Ba}$ & $5,95 \mathrm{Ba}$ & $4,65 \mathrm{Aa}$ & $4,37 \mathrm{Ba}$ & $5,50 \mathrm{Ba}$ & $4,68 \mathrm{Ba}$ \\
\hline A2 & $4,30 \mathrm{Ba}$ & $6,25 \mathrm{Ba}$ & $4,27 \mathrm{Aa}$ & $5,63 \mathrm{Ba}$ & $4,23 \mathrm{Ba}$ & $4,50 \mathrm{Ba}$ \\
\hline A3 & $5,68 \mathrm{Ba}$ & $5,22 \mathrm{Ba}$ & $5,10 \mathrm{Aa}$ & 7,47 Ba & $4,40 \mathrm{Ba}$ & $4,87 \mathrm{Ba}$ \\
\hline \multirow[t]{2}{*}{ A4 } & $14,92 \mathrm{Aab}$ & $16,72 \mathrm{Aa}$ & $8,68 \mathrm{Ab}$ & $11,17 \mathrm{Aa}$ & $15,33 \mathrm{Aa}$ & $16,67 \mathrm{Aa}$ \\
\hline & \multicolumn{6}{|c|}{ V\% } \\
\hline A1 & $27,00 \mathrm{Ba}$ & $37,17 \mathrm{Ba}$ & $31,67 \mathrm{ABa}$ & $29,50 \mathrm{Ba}$ & $33,67 \mathrm{Ba}$ & $32,00 \mathrm{Ba}$ \\
\hline$A 2$ & $27,67 \mathrm{Ba}$ & $38,00 \mathrm{Ba}$ & $27,83 \mathrm{Ba}$ & $34,17 \mathrm{ABa}$ & $29,33 \mathrm{Ba}$ & $29,17 \mathrm{Ba}$ \\
\hline$A 3$ & $36,50 \mathrm{Ba}$ & $34,17 \mathrm{Ba}$ & $31,00 \mathrm{Ba}$ & $38,83 \mathrm{ABa}$ & $29,00 \mathrm{Ba}$ & $31,00 \mathrm{Ba}$ \\
\hline \multirow[t]{2}{*}{ A4 } & $68,50 \mathrm{Aa}$ & $65,33 \mathrm{Aa}$ & $44,33 \mathrm{Ab}$ & $44,83 \mathrm{Ab}$ & $60,33 \mathrm{Aa}$ & $67,33 \mathrm{Aa}$ \\
\hline & \multicolumn{6}{|c|}{$\mathrm{H}+\mathrm{Al}\left(\mathrm{mmol}_{\mathrm{c}} \mathrm{dm}^{-3}\right)$} \\
\hline A1 & $10,67 \mathrm{Aa}$ & $10,17 \mathrm{Aa}$ & $10,17 \mathrm{Aa}$ & 10,17 Aa & $10,67 \mathrm{ABa}$ & $10,00 \mathrm{Aa}$ \\
\hline A2 & $11,00 \mathrm{Aa}$ & $10,17 \mathrm{Aa}$ & $11,00 \mathrm{Aa}$ & $10,67 \mathrm{Aa}$ & $11,00 \mathrm{Aa}$ & $10,50 \mathrm{Aa}$ \\
\hline$A 3$ & $9,83 \mathrm{Aa}$ & $10,00 \mathrm{Aa}$ & $10,67 \mathrm{Aa}$ & $11,17 \mathrm{Aa}$ & $10,67 \mathrm{ABa}$ & $10,67 \mathrm{Aa}$ \\
\hline A4 & $7,50 \mathrm{Bb}$ & 8,67 Aab & $9,33 \mathrm{Aa}$ & $10,33 \mathrm{Ba}$ & 8,67 Bab & $7,50 \mathrm{Bb}$ \\
\hline
\end{tabular}

$\mathrm{S}=$ solo nu; $\mathrm{G}$ = feijāo guandu; $\mathrm{M}=$ mucuna; $\mathrm{B}=$ braquiária; $\mathrm{G}+\mathrm{B}=$ guandu + braquiária; $\mathrm{M}+\mathrm{B}=$ mucuna + braquiária. $\mathrm{A} 1$ = adubação química (NPK); $\mathrm{A} 2$ = adubação orgânica (composto orgânico); A3 = adubação orgânica (composto orgânico) + NPK; A4 = adubaçấo orgânica (composto orgânico) + NPK + calagem.

Para cada cobertura, letras minúsculas iguais na linha indicam que as médias não diferiram entre si, pelo teste de Tukey $(p>0,05)(n=6)$.

Para cada adubaçăo, letras maiúsculas iguais na coluna indicam que as médias năo diferiram entre si, pelo teste de Tukey $(\mathrm{p}>0,05)(\mathrm{n}=6)$. 
Em relação aos atributos físicos (Tabela 4), os resultados mostram que a densidade do solo, a densidade de partículas e a porosidade total não revelaram diferenças significativas entre os tratamentos, constatando que os 15 meses de introdução de vegetação na área não foram suficientes para promover mudanças significativas nesses atributos, mesmo com o uso de gramíneas.

A estrutura do solo desempenha um papel fundamental nas relaçôes entre as fases sólida, líquida e gasosa do solo, de acordo com Silva e Ribeiro (1982); mesmo com o uso de fertilizantes, variedades adaptadas e controles locais, bons resultados só serão atingidos se não houver degradação da estrutura do solo. NASCiMENTO et al. (2005) observaram que em três anos de avaliação em terrenos cultivados com leguminosas, foram mantidas inalteradas as características de densidade do solo, porosidade total, argila em água, estabilidade de agregados do solo.

Quanto à argila, ao silte e à areia total, não houve diferenças significativas entre tratamentos, porém observou-se diferenças entre blocos, revelando grande variação textural no terreno no sentido do escoamento e da sedimentação das partículas, sendo mais arenoso na cabeceira e mais argiloso nas áreas de baixada, conforme se pode observar também em Espíndola et al. (2000).

Em relação ao estabelecimento da vegetação procurou-se identificar quais seriam as espécies que conseguiriam se estabelecer neste tipo de substrato. Pela análise da tabela 5 permitiu-se observar que o solo sem cobertura vegetal proporcionou os piores resultados na avaliação da formação do tapete verde, e somente dentro da adubaçáo química A1 as médias foram iguais. As melhores coberturas foram o feijão guandu, a mucuna preta, o feijão guan$\mathrm{du}+$ braquiaria bumicula e a mucuna preta + braquiaria bumidicula na adubação orgânica + adubação química + calagem; os tratamentos com a utilização da mucuna preta e do feijão guandu + braquiaria humidicula proporcionaram bom recobrimento do solo.

Mesmo não diferindo significativamente entre si, observa-se maior tendência obtida com a adubação orgânica, a adubação orgânica + adubação química e a adubação

Tabela 4. Densidade do solo, densidade de partículas, porosidade total, areia, silte e argila em rejeito arenoso com diferentes coberturas vegetal e tipos de adubaçáo

\begin{tabular}{|c|c|c|c|c|c|c|}
\hline \multirow{2}{*}{ Adubação } & \multicolumn{6}{|c|}{ Densidade do solo $\left(\mathrm{g} \mathrm{cm}^{-3}\right)$} \\
\hline & $\mathbf{S}$ & G & M & B & $\mathbf{G}+\mathbf{B}$ & $M+B$ \\
\hline A1 & $1,46 \mathrm{Aa}$ & $1,43 \mathrm{Aa}$ & $1,42 \mathrm{Aa}$ & $1,45 \mathrm{Aa}$ & $1,45 \mathrm{Aa}$ & $1,45 \mathrm{Aa}$ \\
\hline$A 2$ & $1,44 \mathrm{Aa}$ & $1,41 \mathrm{Aa}$ & $1,45 \mathrm{Aa}$ & $1,41 \mathrm{Aa}$ & $1,33 \mathrm{Aa}$ & $1,47 \mathrm{Aa}$ \\
\hline A3 & $1,46 \mathrm{Aa}$ & $1,46 \mathrm{Aa}$ & $1,42 \mathrm{Aa}$ & $1,44 \mathrm{Aa}$ & $1,48 \mathrm{Aa}$ & $1,41 \mathrm{Aa}$ \\
\hline \multirow[t]{2}{*}{ A4 } & $1,40 \mathrm{Aa}$ & $1,43 \mathrm{Aa}$ & $1,33 \mathrm{Aa}$ & $1,35 \mathrm{Aa}$ & $1,43 \mathrm{Aa}$ & $1,40 \mathrm{Aa}$ \\
\hline & \multicolumn{6}{|c|}{ Densidade de partículas $\left(\mathrm{g} \mathrm{cm}^{-3}\right)$} \\
\hline A1 & $2,77 \mathrm{Aa}$ & $2,73 \mathrm{Aa}$ & $2,72 \mathrm{Aa}$ & $2,76 \mathrm{Aa}$ & $2,73 \mathrm{Aa}$ & $2,74 \mathrm{Aa}$ \\
\hline $\mathrm{A} 2$ & $2,70 \mathrm{Aa}$ & $2,74 \mathrm{Aa}$ & $2,75 \mathrm{Aa}$ & $2,70 \mathrm{Aa}$ & $2,75 \mathrm{Aa}$ & $2,71 \mathrm{Aa}$ \\
\hline A3 & $2,68 \mathrm{Aa}$ & $2,75 \mathrm{Aa}$ & 2,74 Aa & $2,75 \mathrm{Aa}$ & $2,70 \mathrm{Aa}$ & $2,72 \mathrm{Aa}$ \\
\hline \multirow[t]{2}{*}{ A4 } & $2,40 \mathrm{Aa}$ & $2,73 \mathrm{Aa}$ & $2,73 \mathrm{Aa}$ & $2,72 \mathrm{Aa}$ & $2,71 \mathrm{Aa}$ & $2,71 \mathrm{Aa}$ \\
\hline & \multicolumn{6}{|c|}{ Porosidade total (\%) } \\
\hline A1 & $47,31 \mathrm{Aa}$ & 47,56 Aa & $47,89 \mathrm{Aa}$ & $47,38 \mathrm{Aa}$ & 46,92 Aa & 47,09 Aa \\
\hline$A 2$ & $46,53 \mathrm{Aa}$ & $48,40 \mathrm{Aa}$ & $47,09 \mathrm{Aa}$ & $47,87 \mathrm{Aa}$ & $51,45 \mathrm{Aa}$ & 45,79 Aa \\
\hline \multirow[t]{2}{*}{ A4 } & $52,40 \mathrm{Aa}$ & $47,37 \mathrm{Aa}$ & $51,31 \mathrm{Aa}$ & $5045 \mathrm{Aa}$ & $47,41 \mathrm{Aa}$ & 48,39 Aa \\
\hline & \multicolumn{6}{|c|}{ Argila (g kg $\left.{ }^{-1}\right)$} \\
\hline A1 & $81,7 \mathrm{Aa}$ & $80,0 \mathrm{Aa}$ & $81,7 \mathrm{Aa}$ & 75,0 Aa & $80,0 \mathrm{Aa}$ & $81,7 \mathrm{Aa}$ \\
\hline$A 2$ & $86,7 \mathrm{Aa}$ & $76,7 \mathrm{Aa}$ & $85,0 \mathrm{Aa}$ & $73,3 \mathrm{Aa}$ & $90,0 \mathrm{Aa}$ & $83,3 \mathrm{Aa}$ \\
\hline$A 3$ & $68,3 \mathrm{Aa}$ & $80,0 \mathrm{Aa}$ & $90,0 \mathrm{Aa}$ & $83,3 \mathrm{Aa}$ & $81,7 \mathrm{Aa}$ & $81,7 \mathrm{Aa}$ \\
\hline \multirow[t]{2}{*}{ A4 } & $68,2 \mathrm{Aa}$ & $95,0 \mathrm{Aa}$ & $85,0 \mathrm{Aa}$ & $93,3 \mathrm{Aa}$ & $85,0 \mathrm{Aa}$ & $98,3 \mathrm{Aa}$ \\
\hline & \multicolumn{6}{|c|}{ Silte $\left(\mathrm{g} \mathrm{kg}^{-1}\right)$} \\
\hline A1 & $118,3 \mathrm{Aa}$ & $166,7 \mathrm{Aa}$ & $145,0 \mathrm{Aa}$ & $128,3 \mathrm{Aa}$ & $141,7 \mathrm{Aa}$ & $126,7 \mathrm{Aa}$ \\
\hline A2 & $146,7 \mathrm{Aa}$ & $126,7 \mathrm{Aa}$ & $140,0 \mathrm{Aa}$ & $156,7 \mathrm{Aa}$ & $160,0 \mathrm{Aa}$ & $143,3 \mathrm{Aa}$ \\
\hline A3 & $125,0 \mathrm{Aa}$ & $141,7 \mathrm{Aa}$ & $141,7 \mathrm{Aa}$ & $145,0 \mathrm{Aa}$ & $140,0 \mathrm{Aa}$ & $140,0 \mathrm{Aa}$ \\
\hline \multirow[t]{2}{*}{ A4 } & $149,2 \mathrm{Aa}$ & $140,0 \mathrm{Aa}$ & $138,3 \mathrm{Aa}$ & $170,0 \mathrm{Aa}$ & $151,7 \mathrm{Aa}$ & $145,0 \mathrm{Aa}$ \\
\hline & \multicolumn{6}{|c|}{ Areia total $\left(\mathbf{g ~ k g}^{-1}\right)$} \\
\hline A3 & $798,3 \mathrm{Aa}$ & $768,3 \mathrm{Aa}$ & $768,3 \mathrm{Aa}$ & 771,7 Aa & $781,7 \mathrm{Aa}$ & $778,3 \mathrm{Aa}$ \\
\hline A4 & $781,4 \mathrm{Aa}$ & $781,7 \mathrm{Aa}$ & $793,3 \mathrm{Aa}$ & $736,7 \mathrm{Aa}$ & $765,0 \mathrm{Aa}$ & $758,3 \mathrm{Aa}$ \\
\hline
\end{tabular}

$\mathrm{S}=$ solo nu; $\mathrm{G}$ = feijăo guandu; $\mathrm{M}=$ mucuna; $\mathrm{B}=$ braquiária; $\mathrm{G}+\mathrm{B}=$ guandu + braquiária; $\mathrm{M}+\mathrm{B}=$ mucuna + braquiária. A1 = adubaçăo química (NPK); 2 = adubaçăo orgânica (composto orgânico); A3 = adubação orgânica (composto orgânico) + NPK; A4 = adubaçáo orgânica (composto orgânico) + NPK + calagem.

Para cada cobertura, letras minúsculas iguais na linha indicam que as médias năo diferiram entre si, pelo teste de Tukey $(\mathrm{p}>0,05)(\mathrm{n}=6)$.

Para cada adubaçáo, letras maiúsculas iguais na coluna indicam que as médias não diferiram entre si, pelo teste de Tukey $(\mathrm{p}>0,05)(\mathrm{n}=6)$. 
Tabela 5. Notas atribuídas à cobertura vegetal das espécies plantadas e da vegetação espontânea em rejeito arenoso com diferentes coberturas vegetal e tipos de adubação

\begin{tabular}{|c|c|c|c|c|c|c|}
\hline \multirow{3}{*}{ Adubação } & \multicolumn{6}{|c|}{ Cobertura verde } \\
\hline & 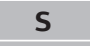 & G & $M$ & B & $G+B$ & $M+B$ \\
\hline & \multicolumn{6}{|c|}{ Notas } \\
\hline A1 & $1,7 \mathrm{Aa}$ & 3,6Aa & 4,1aAa & 3,5Aa & $2,4 \mathrm{Aa}$ & $3,5 \mathrm{Aa}$ \\
\hline A2 & $2,1 \mathrm{Aa}$ & 4,0 Bab & $4,9 \mathrm{bAa}$ & 4,4bABab & $5,3 \mathrm{ABb}$ & $5,1 \mathrm{Bb}$ \\
\hline A3 & $2,2 \mathrm{Aa}$ & $6,5 \mathrm{Bb}$ & $5,0 \mathrm{bAa}$ & $6,1 \mathrm{Bb}$ & $5,5 \mathrm{ABb}$ & $6,1 \mathrm{Bb}$ \\
\hline A4 & $2,3 \mathrm{Aa}$ & $5,3 \mathrm{Bbc}$ & $7,4 \mathrm{Bbc}$ & $4,9 \mathrm{bABab}$ & $7,5 \mathrm{Bc}$ & $6,5 \mathrm{Bbc}$ \\
\hline
\end{tabular}

$\mathrm{S}=$ solo nu; $\mathrm{G}=$ feijăo guandu; $\mathrm{M}=$ mucuna; $\mathrm{B}=$ braquiária; $\mathrm{G}+\mathrm{B}=$ guandu + braquiária; $\mathrm{M}+\mathrm{B}=$ mucuna + braquiária. $\mathrm{A} 1=$ adubação química $(\mathrm{NPK}) ; \mathrm{A} 2$ = adubação orgânica (composto orgânico); A3 = adubação orgânica (composto orgânico) + NPK; A4 = adubação orgânica (composto orgânico) + NPK + calagem.

Para cada cobertura, letras minúsculas iguais indicam que as médias năo diferiram entre si, pelo teste de Tukey $(\mathrm{p}>0,05)(\mathrm{n}=6)$.

Para cada adubação, letras maiúsculas iguais indicam que as médias não diferiram entre si, pelo teste de Tukey $(\mathrm{p}>0,05)(\mathrm{n}=6)$.

orgânica + adubação química + calagem. Cravo e SMITH (1997), estudando a produção de grãos em áreas desmatadas na região amazônica, observaram que a ausência da calagem promoveu redução no rendimento da produção de soja entre o $1 .^{\circ}$ e o $2 .^{\circ}$ ano de cultivo evidenciando a importância da calagem em solos tropicais na região amazônica.

Pelos resultados, pode-se observar que a adubação verde, mesmo não diferindo entre si, principalmente utilizando-se mucuna preta e feijão guandu + braquiária, juntamente com a adubação orgânica proporcionaram boa tendência de cobertura do solo, o que poderá modificar de modo positivo algumas propriedades do solo/substrato em questão. Porém, os 15 meses decorridos, mesmo com o estabelecimento da vegetação, não foram suficientes para promover alteraçôes nas propriedades físicas do solo. As propriedades químicas, principalmente $\mathrm{pH}$, $\mathrm{Ca}, \mathrm{Mg}, \mathrm{T}, \mathrm{SB}$ e V tiveram alterações em decorrência da adubação química e da calagem. SANChes (2002) observou que a mucuna preta proporcionou bom desenvolvimento em solos degradados pela mineração de cassiterita, e uma dose de $100 \mathrm{mg} \mathrm{kg}^{-1}$ de $\mathrm{P}$ proporcionou bons resultados quando a planta foi submetida à inoculaçãoo com Bradyrhizobium. Nogueira (2000) observou que o capim Tanzânia proporcionou boa produção de massa seca em condições similares, podendo também esse tipo de cobertura ser utilizado como tapete verde.

Assim, quando se deseja um rápido recobrimento do terreno e produção de matéria orgânica, pode-se optar pelo plantio de mucuna ou feijão guandu + braquiária com adubação orgânica + adubação química + calagem. Porém outras opçóes podem ser usadas dependendo de alguns fatores que influirão na tomada de decisão, como custo, disponibilidade de materiais e sementes, tempo, etc. Salienta-se que este é um trabalho preliminar e que novas avaliaçóes serão realizadas o que proporcionará uma continuidade na análise e discussão sobre cobertura vegetal em solos degradados por explotação mineral. LUNARDi NeTO et al. (2008) observaram que a correlação do solo reconstituído após exploração de carvão deve ser acompanhada da introdução de espécies que cobrem o solo e pela adição de matéria orgânica para fornecer os mecanismos de floculação da matéria orgânica e estabilização dos agregados.
Considerando os aspectos metodológicos envolvidos e a análise dos dados, os 15 meses decorridos entre o plantio e a primeira amostragem de solo não foram suficientes para promover alteraçôes em algumas propriedades do solo avaliadas. As características químicas foram as únicas em que se notaram diferenças significativas entre os tratamentos. A própria adubação química, orgânica e a calagem contribuíram para essas diferenças e nos tratamentos que receberam calcário, constatou-se uma série de melhorias ao substrato como aumento no $\mathrm{pH}$, nos teores de $\mathrm{Ca}, \mathrm{Mg}$, na CTC, SB e V\% e uma diminuição no $\mathrm{H}+\mathrm{Al}$. No entanto, quando se analisa o teor de matéria orgânica, os atributos físicos e os aspectos biológicos estudados, estes não revelam diferenças entre os tratamentos, mesmo naqueles onde a adubaçáo foi intensiva associada ao plantio de adubo verde e a introdução de espécies florestais.

Dessa maneira, visando obter respostas mais conclusivas, será necessário que se realizem novas amostragens em um intervalo maior de tempo, pois o estabelecimento de um -horizonte A determina o sucesso e a sustentabilidade do processo de recuperaçáo de solos degradados.

\section{CONCLUSÕES}

1. Os tratamentos com adubação química, orgânica e calagem, bem como o plantio de adubos verdes e gramíneas como cobertura, alteraram a fertilidade do solo mas não modificaram o teor de matéria orgânica e propriedades físicas nos 15 meses estudados;

2. Há necessidade de um tempo maior de utilizaçáo da adubação para que se obtenha resultados significativos para estes parâmetros, quando se pretende a recuperaçáo de solos degradados por atividades minerarias pelo uso de diferentes formas de adubação.

\section{AGRADECIMENTOS}

À Fundação de Amparo à Pesquisa do Estado de São Paulo (FAPESP), a Brascan e ao Instituto Brasileiro do Meio Ambiente e Recursos Minerais Renováveis (IBAMA). 


\section{REFERÊNCIAS}

BANZATTO, D.A.; KRONKA, S.N. Experimentação agrícola. 2.ed. Jaboticabal: Funep, 1992. 247p.

COCHURANE, T.T.; SANCHES, P.A. Land resources, soils properties and their management in the Amazon region: a state of knowledge report. In: HECHT, S.B. (Ed.). Amazon land use research. Cali: CIAT, 1982. p.138-209.

COSTA, S.; ZOCCHE, J.J. Fertilidade de solos construídos em áreas de mineração de carvão na região sul de Santa Catarina. Revista Árvore, v.33, p.205-214, 2009.

CRAVO, M.S.; SMYTH, T.J. Manejo sustentado da fertilidade de um Latossolo da Amazônia central sob cultivos sucessivos. Revista Brasileira de Ciência do Solo, v.27, p.607-616, 1997.

EMBRAPA. Centro Nacional de Pesquisa de Solos. Sistema brasileiro de classificação de solos. Brasília: Embrapa Produção de Informação, 1999. 412p.

ESPÍNDOLA, C.R.; MELO, W.J.; LONGO, R.M. Forest and soil degradation by tin minering. In: SYMPOSIUM MANAGING FOREST SOILS FOR SUSTAINABLE PRODUCTIVITY, 2000, Vila Real (Portugal). Abstracts... Vila Real: SPCS, 2000, p.215-214.

FRANÇA, J.T. Estudos da sucessão secundária em áreas contíguas a mineração de cassiterita na Floresta Nacional do Jamari-RO. 1991, 169p. Dissertação (Mestrado- Curso de Engenharia Florestal) Escola Superior de Agricultura/USP, Piracicaba.

FONTES, M.P.F. Estudo pedológico reduz impacto da mineração. Revista Cetesb de Tecnologia e Ambiente, v.5, p. 58-61, 1991.

GRIFFITH, J.J. Recuperação conservacionista da superfície de áreas mineradas: Uma revisão de literatura. Viçosa: Sociedade de Investigaçóes Florestais, 1980. 106p. (Boletim Técnico n. 79)

GRIFFITH, J.J.; DIAS, L.E.; JUCKSON. Recuperação de áreas degradadas usando vegetaçáo nativa. Saneamento Ambiental, v.6, p.28-37, 1996.

KIEHL, E.J. Manual de edafologia: relação solo-planta. São Paulo: Ceres, 1979. 262p.

LONGO, R.M.; RIBEIRO, A.I.; MELO, W.J. Caracterização física e química de áreas mineradas pela extração de cassiterita. Bragantia, v.64, p.101-107, 2005.

LUNARDI NETO, A.; ALBUQURQUE, J.A.; ALMEIDA, J.A.; MAFRA, A.L., MEDEIROS, J.C. Atributos físicos do solo em área de mineração de carvão influenciados pela correção de acidez, adubação orgânica e revegetação. Revista Brasileira de Ciência do Solo, v.32, p.1379-1388, 2008.
NASCIMENTO, J.T.; SILVA, I.F;; ROBERVAL, D.S.; SILVA NETO, L.F. Efeito de leguminosas bis atributos físicos e carbono orgânico de um Luvissolo. Revista Brasileira de Ciência do Solo, v.29, p.825-831, 2005.

NOGUEIRA, A. E. Capim Tanzânia para a recuperação de áreas degradadas pela mineração de cassiterita na regiáo amazônica. 2000. 112p. Trabalho de conclusão de curso (Curso de Agronomia),Universidade Estadual Paulista, Jaboticabal (SP).

OLIVEIRA, C.V. Micorrização, compactação e fósforo no crescimento de leguminosas arbóreas em solo degradado 1995. 54p. Dissertação (Mestrado - Curso de Agronomia) - Universidade Federal de Lavras.

RADAMBRASIL. Folha SC-20. Porto Velho. Rio de Janeiro: Departamento Nacional de Produçáo Mineral, 1978. 661p. (Levantamento de Recursos Naturais, 16)

RAIJ, B.van; ZULLO, M.A.T. Análise química do solo para fins de fertilidade. Campinas: Fundaçáo Cargill, 1987. 170p.

RAIJ, B. van. Fertilidade do solo e adubação. Piracicaba: Ceres/ Potafos, 1991. 343p.

RAIJ, B. van; CANTARELLA, H. QUAGGIO, J.A. FURLANI, A.M.C. (Ed.). Recomendações de adubação e calagem para o Estado de São Paulo. Campinas: Instituo Agronômico, 1996. 285p.

SANCHES, A.C. Adubação fosfatada e inoculação de leguminosas com Bradyrhizobium na recuperaçáo de solo degradado pela mineração de cassiterita na região amazônica. 2002. 96p. Tese (Doutorado-Curso de Agronomia) - Universidade Estadual Paulista, Jaboticabal (SP).

SILVA, K.E. Adequação química e física para a revegetação de um rejeito de mineração de ferro. 1993. 80 p. Dissertação (Mestrado) Universidade Federal de Viçosa (MG)

SILVA, M.S.L.; RIBEIRO, M.R. Influência do cultivo contínuo de cana-de-açúcar nas propriedades morfológicas e físicas de solos argilosos de tabuleiro no estado de Alagoas. Revista Brasileira de Ciência do Solo, v.16, p.397-402, 1992.

SILVA, S.R.; PROCÓPIO, S.O.; QUEIROZ, T.F.N.; DIAS, L.E. Caracterização de rejeito de mineração de ouro para avaliação de solubilidade de metais pesados e arsênio e revegetação local. Revista Brasileira de Ciência do Solo, v.28, p-189-196, 2004.

WILLIAMS, D.D.; BUGIN, A.; REIS, J.L.B.C. Manual de Recuperação de Áreas degradadas pela mineração: técnicas de revegetação. Brasília: Instituo Brasileiro do Meio Ambiente e dos Recursos Naturais Renováveis - IBAMA, 1990. 96p. 